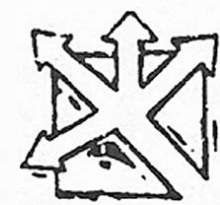

http://doi.org/10.15359/ree.2004-6.10

Educación Superior

\title{
LAS NECESIDADES EDUCATIVAS ESPECIALES DE LOS ESTUDIANTES: UN ASUNTO DE TODOS
}

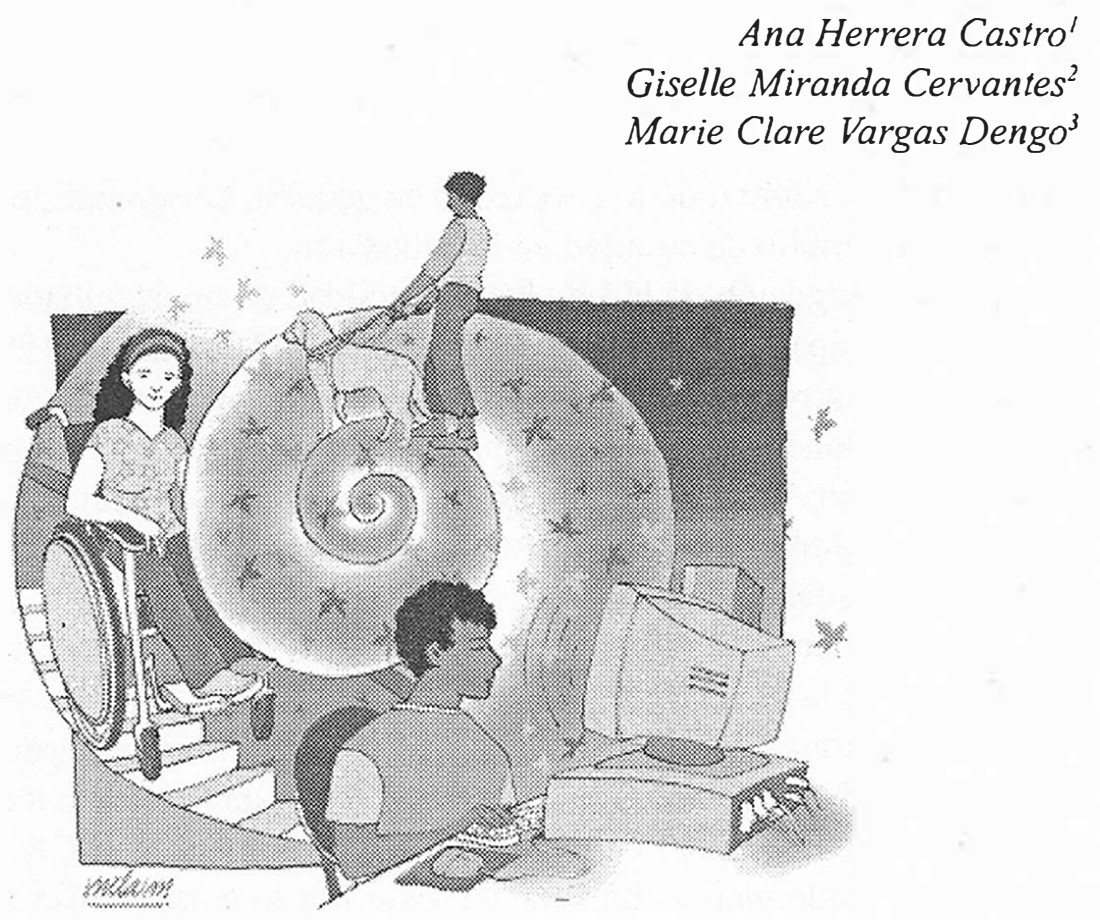

1 Licenciada en Educación Especial con énfasis en Retardo Mental, Universidad Nacional, Egresada de la Maestría en Integración de personas con discapacidad, Universidad de Salamanca, España. Actualmente es Directora de la División de Educación Básica del CIDE, Universidad Nacional, donde se ha desempeñado como docente, extensionista e investigadora desde 1990.

2 Licenciada en Psicología, y Egresada de la Maestría en Psicología, Universidad de Costa Rica. De 1992 a 1999 coordinó la Carrera de Educación Especial y desde el 2002 coordina la Carrera de Enseñanza del Inglés para l y II Ciclos en la División de Educación Básica del CIDE, Universidad Nacional, donde se ha desempeñado como docente e investigadora desde 1990.

3 Master of Arts in Bilingual Special Education, George Washington University, USA. Desde el 2000 es docente"en la Universidad Nacional y actualmente coordina el Proyecto Una Educación para todos los alumnos de la Universidad Nacional que desarrolla la carrera de Educación Especial de la División de Educación Búsica. 
Comprender los beneficios de educar a una población diversa, significa considerar la di versidad como valor, citando a Devalle y Vega, (1999): "La diversidad considerada como valor implica orientar la educación hacia los principios de igualdad, justicia y libertad, y todo ello para establecer un compromiso permanente con las culturas y grupos minoritarios" (p. 39). Asegurar la accesibilidad a la educación superior de los estudiantes con necesidades
El presente articulo describe la experiencia generada desde la carrera de Educación Especial, asi como los apontes que a lo largo de seis años ha brindado el proyecto Una educación para tolos los alumnos de la Universidad Nacional, en la atención de las necesidades educativas especiales de los estudiantes. Contemplando el valor positivo de la diferencia, se incluyen algunas percepciones de los diferenres actores sociales de este proyecto como son: un estudiante con discapacidad, una estudianre de horas colaboración. una essudianse asis. tente y una académica, con el fin de compartir su vivencia con los leciores.

educativas especiales, derivadas o no éstas de una discapacidad, beneficia en última instancia no solo a la población con discapacidad, sino a la comunidad universitaria y, sobre todo a la sociedad en general, comprendido esto dentro de un concepto amplio de equidad en la educación.

La promulgación de la Ley 7600, Igualdad de oportunidades para las personas con discapacidad en Costa Rica (Asamblea Legislativa, 1996), marca una etapa en el desarrollo social de nuestro país, en la que se toma conciencia, tanto en las instituciones públicas como privadas, de cumplir con los requerimientos de la legislación, y por definir qué hacer y cómo hacerlo. Esta inquictud germina en muchos ámbitos profesionales, generando iniciativas positivas para la población con discapacidad.

Este es el caso de la Universidad Nacional (UNA) y, en particular, de las académicas de la carrera de Educación Especial del Centro de Investigación y Docencia en Educación (CIDE); al procurar la inserción y pasticipación de las personas con discapacidad. Aunque una idea de esta índole no tiene por qué ser exclusiva de una facultad de educación, es de reconocer que la trayectoria de este Centro académico se ha caracterizado por el compromiso social y por la identificación de aquellas situaciones que puedan obstaculizar el proceso de aprendizaje de los alumnos.

En las últimas décadas se ha tratado de atender a la población con discapacidad, desde distintos paradigmas y se han utilizado términos como segregación, integración, atención a las necesidades educativas especiales, inclusión y atención a la diversidad, entre otros. No obstante, la dimensión del asunto va mucho más allá de algo meramente conceptual, por lo que se hace necesario aclarar algunos de estos conceptos para comprender a qué nos referimos cuando 
Description of the experience bencerented as Universidad Nacional s Special Edecasion major and of the contributions muele by project "UNA S Edurcestion for All" during the last sir yeurs. The project has heolped ilee ssudents of this university to satisfy their special needs. In oreler to confemplase she positive value posed by differences in people. we in. clude here the opinions of several social a handicap student. a sfuclent from the pro. giam" "Collaboration Hours". an assistant student and a scholar: It is our desire 10 shure elecir experiences with our readers. agents that have played a role in the project:

hablamos de necesidades educativas especiales. Dentro de las varias acepciones, Brennan (1988), por ejemplo, se refiere a este concepto indicando que:

"Hay una necesidad educativa especial cuando una deficiencia (fisica. sensorial, intelectual, emocional, social o cualquier combinación de éstas) afccta el aprendizaje hasta tal punto que son necesarios algunos o todos los cambios especiales al currículo, al currículo especial o modificado, o algunas condiciones de aprendizaje especialmente adaptadas para que el alumno sea cducado adecuada y eficazmente. La necesidad puede presentarse en cualquier punto de un continuo que va desde la leve hasta la aguda; puede ser permanente o una fase temporal en el desarrollo del alumno". (p. 36).

A pesar de ser esta una definición amplia, centra el problema en la deficiencia del sujeto y no en la deficiencia del entomo a ajustarse a las condiciones particulares de cada deficiencia.

Costa Rica, a partir de 1994 y después de participar en la Conferencia Mundial de Salamanca como muchos otros paises, asume desde otra perspectiva, el concepto de necesidades educativas especiales para referirse a las necesidades que presentan algunas personas en el proceso de aprendizaje. La connotación heredada de paradigmas anteriores, como ya se mencionó, enfatiza "las dificultades que presenta el estudiante en su aprendizaje" y no "las condiciones personales o culturales de estilo y ritmo de aprendizaje para las que el curriculum promedio resulta desfasado o insuficiente" (Meléndez, 2000). Desde esta otra perspectiva, el concepto necesidades educativas especiales adquiere un carácter interactivo (sujeto-entomo) en el que las necesidades educativas especiales no dependen de las limitaciones personales sino de aquellas caracteristicas del contexto que pueden resultar barreras para el aprendizaje.

Por tanto, se hace necesario eliminar las barreras que el entorno impone a los sujetos que aprenden, sean éstas físicas, sociales o actitudinales. Dos estrategias que pueden contribuir a lo anterior, son: garantizar la participación plena de las personas con discapacidad en todos los aspectos y decisiones que las involucre 
e incidan en su desarrollo, así como el fortalecimiento de proyectos de investigación dirigidos a identificar obstáculos y a eliminar barreras.

\section{Origen del proyecto: una educación para todos los alumnos de la Univer- sidad Nacional}

Con la intención de identificar un momento concreto en la historia de la Universidad Nacional en cl cual se oficializan algunas iniciativas tendientes a la atención sistemática de estudiantes con discapacidad, es importante mencionar a 1992 como el año en el cual el Consejo Universitario solicita a la Vicerrectoría de Vida Estudiantil establecer las directrices que faciliten que estos cstudiantes dispongan de alternativas válidas para realizar adccuadamente sus estudios en lat institución (SCU-1893, 1992).

Es de esta manera que en 1994, y con la participación de un cquipo multidisciplinario, constituido por profesionales de diferentes árcas de la Universidad Nacional como la carrera de Educación Especial, el Departamento de Orientación y Psicologia, cl Área de Plancamicnto Espacial, el Depara amento de Biencstar Estudiantil, la Viceriectoría Académica y cl Departamento de Sallud; conjuntamente con cstudiantes con discapacidad en representación de la Fedcración de Estudiantes (FEUNA) y un representante de la Asociación Costarricense de Lisiados, se elabora y aprueba cl Plan de at ención global del estudiante con discapacidad (Rodriguez ct al., 1994) como forma de garantizar cl cumplimiento del principio de igualdad de oportunidades para todas las personas que deseen ingresar y permanecer en la Universidad Nacional.

En forma paralela a los procesos institucionales antes mencionados, en la cartrera de Educación Especial del Centro de Investigación y Docencia en Educación (CIDE), ya se concretaban acciones tendientes a ofrecer apoyos que facilitaran el proceso de aprendizaje de algunos cstudiantes matriculados en la Universidad Nacional, no obstantc estas iniciativas respondían más bien al compromiso de sus académicas que por mucho ticmpo se tradujo en trabajo pionero, voluntario y extracurricular, pero silencioso, y del cual conocieron únicamente los estudiantes con discapacidad que recibieron los bencficios.

Afortunadamente para la institución, es a partir de cstas cxperiencias que en 1999 la carrera de Educiación Especial asume el reto y formula el proyecto: una educación para todos los alumnos de la Universidad Nacional (Herrera, Miranda y Zúñiga, 1998) cuyo objetivo fundamental durante los scis años que hat estado vigente, ha sido ofrecer una respucsta adecuada a las necesidades educativas especiales de los estudiantes de la UNA que, por su discapacidad, 
enfrentan alguna condición de desventaja y requicren de apojos específicos para compensarlas y disfrutar de todas las oportunidades que la Universidad ofrecc.

Este proyecto, en congruencia con lo que cstipula cl programa "Educación para Todos" (1990), en cl cual la UNESCO establece el derecho a una educación adaptada a las necesidades de cada estudiante, independicntemente de la magnitud de la deficiencia o necesidad particular (Van Sicenlandt, 1991), desde un modelo que promueve el derccho a la cducación de grupos particulares y en un esfucrzo por atender las necesidacies educativas de los estudiantes con discapacidad; procura of recer alternativas que aseguren al cstudiante la accesibilidad en los distintos cursos de la cartera universitaria que han optado seguir:

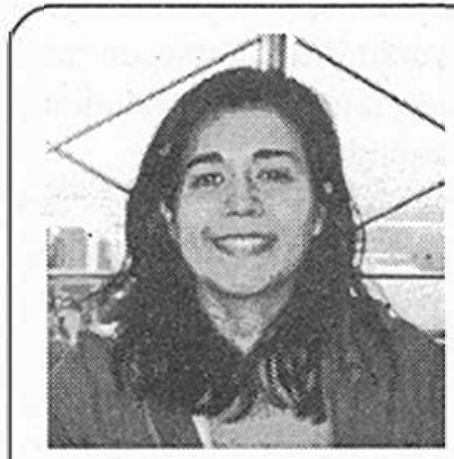

"En un momento en el que es indispensable reconocer la diversidad en el aula universitaria, el proyecto Una educación para todos los alumnos de la UNA sicnta un precedente en la atención de una parte de esta diversidad, fortaleciendo la igualdad de oportunidades en cl aula y brindando de una forma certera los instumentos para atender a la población con discápácidad.

El proyecto, además, de proporcionar heramientas a los cstudiantes, realiza una labor sumamente importante de apoyo al docente universitario, pucs ayuda a que los profesores reciban información sobre cl estudiante con discapacidad y la atención que necesitan en el salón de clases. Esto hace que la comunidad de profesores se vaya sensibilizando hacia esta población y poco a poco incorporen en sus lecciones las adecuaciones curriculares pertinentes.

El componente humano del proyecto es uno de los recursos que más lo han fortalccido, ejemplo de csto son los estudiantes que cumplen horas colaboración, quienes trabajan al lado de los estudiantes inscritos en el proyecto realizando una labor importante, llena de experiencias diversas y fructíferas independicntemente de cual sca su carrera de estudios".

Licda. Erika Vásguez Salazar Académica del Proyecro

Las académicas responsables de csic proyecto, conocedoras de que la atcnción a las necesidades educativas de los cstudiantes es fundamentalmente un "asunto de todos", proponen of recer altemativas dirigidas a los docentes de la 
Universidad Nacional que atienden en sus aulas a estudiantes con discapacidad, mediante procesos de asesoramiento y de apoyo que les actualicen y capaciten para realizar sus intervenciones pedagógicas de manera más eficiente y en congruencia con los principios de solidaridad que rigen el quehacer institucional (Universidad Nacional, 1993). Igualmente, dicho proyecto pretende ganar un espacio académico en el cual se genere el intercambio de experiencias entre profesores, a fin de determinar los puntos de encuentro en las estrategias que individualmente, pero en forma exitosa, han desarrollado estos profesionales para atender en sus cursos a estudiantes con discapacidad.

Las interacciones personales son también tema de interés del proyecto Una educación para todos los alumnos de la UNA y, por tanto, se propone conocer las preocupaciones, las divergencias y hasta los conflictos que se podrian generar a partir del intercambio que, dentro del aula universitaria, establecen profesor y alumno, a fin de identificar los apoyos requeridos, tanto por los estudiantes con discapacidad, como por los profesores que los atienden.

Con el propósito de promover la atención a las necesidades educativas espcciales de los estudiantes y de facilitar su proceso de aprendizaje, el proyecto promueve vínculos con las distintas Unidades Académicas, procurando una participación cada vez más comprometida. La apertura de los directores, subdirectores y de la mayoría de los profesores, ha sido sumamente positiva, como lo ha sido la colaboración que se recibe de otras instancias como la Oficina de Registro, el Departamento de BienestarEstudiantil, el Departamento de Orientación y Psicología, la Biblioteca García Monge, el Área de Planeamiento Espacial y la Comisión institucional para la atención del estudiante con discapacidad de la Vicerrectoría de Vida Estudiantil.

Otra de las metas de este proyecto, es establecer mecanismos eficientes que permitan a la comunidad universitaria mantenerse actualizada en temas relacionados con discapacidad como: el derecho de los estudiantes con discapacidad a recibir la mejor educación posible, así como la obligación que la Universidad Nacional, como institución del Estado, tiene de promover y garantizar una educación en el marco de los principios de igualdad y equidad.

Una nueva iniciativa gestada desde la carrera de Educación Especial y que ha beneficiado a la población con discapacidad de la Universidad Nacional, fue lograr el otorgamiento de recursos económicos para ejecutar la propuesta UNA: educación de calidad (Herrera y Miranda, 2001) en el Concurso Anual para la distribución del Fondo Institucional de Desarrollo Académico (FIDA). Con la obtención de este financiamiento, se logró adquirir equipo de Apoyo Tecnológico Adaptado entre el cual se incluye computadoras, sofiware adaptado (programa JAWS), escáners, impresoras Braille y grabadoras, entre otros, ubicados en 
el edificio del CIDE, específicamente en la sala del proyecto Una educación para todos los alumnos de la UNA y en el Centro de Información y Documentación en Educación, Niñez, Adolescencia y Familia (CIDENAF). Por olra parte, en coordinación con la Biblioteca Joaquín García Monge y con la Comision institucional para la atención al estudiante con discapacidad, este proyecto ha puesto a la disposición de los estudiantes un equipo de apoyo tecnológico, que a futuro pretende constituirse en la Sala de Apoyo Tecnologico Adaptado (SATA) que ofrezca servicios de apoyo en lo relativo al acceso a la información y a la comunicación.

\section{Un largo camino}

El recuento de muchas de las iniciativas que nacieron a partir del compromiso de los mismos académicos de la Universidad Nacional para atender las neccsidades educalivas de sus estudiantes, se hace de manera rápida en este artículo y básicamente con el interés de contextualizar al lector. No obstante, estamos seguras de que son muchas las iniciativas que día a día han surgido, surgen y surgirán a partir del trabajo académico de profesores comprometidos con la alención de lodos sus estudiantcs.

Es por esto último que, al volver la vista atrás, miramos un largo camino recorrido en el cual han existido muchas sallisfacciones pero tambićn muchos contratiempos, que lejos de rendirnos, más bien nos han motivado para seguir luchando por los derechos de quiencs, por la intolerancia o por la ignorancia de sus profesores, en muchas ocasiones han visto truncados sus deseos de csludiar en una Universidad que desde su creación se autodenomino la Universidad Necesaria.

$\Lambda$ lo largo del tiempo, proyecto Una cducación para todos los alumnos de la UNA de la carrera de Educación Especial, ha construido cspacios importantes de encuentro, de reflexion y de aprendizaje para diferentes seclores de la comunidad universitaria: académicos, administrativos, cstudiantes con discapacidad y estudiantes que participan en cl proyecto (asistentcs y horas colaboración). 


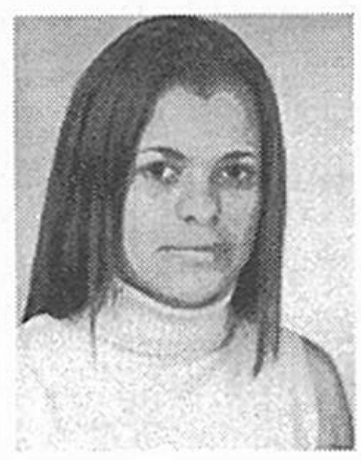
cesidad cducativa cspecial alcancen su sucño de ser profesionales y micmbros activos de la socicdad".

"Comu cstudiante asistente al proyecto Una educación para todos los alumnos de la UNA, he podido vivenciar la puesta en marcha de una educación en igualdad de condiciones. La experiencia que hasta el momento he vivido, me ha permitido entender, pero sobre todo apiender, que con deseos de ayudar $\mathrm{c}$ informar se puede alcanzar esta igualdad. Como estudiante y como futura docente de educación especial, considero que cl proyecto es un camino para que aquellos que presentan alguna nc-

Mauren Angulo Ramire: Essudiante asistense

Entre los años 2002 y 2003, con la participación comprometida de representantes de distintas instancias universitarias, el proyecto, en coordinación con la Comisión institucional para la atención del estudiante con discapacidad y el apoyo de la Vicerrectoría de Vida Estudiantil, organizan talleres para formular las políticas institucionales en materia de discapacidad, hoy en espera de la aprobación del Consejo Universitario. Será de impacto institucional la aprobación de estas políticas que orientarán el quehacer de la UNA en todo lo concerniente a discapacidad.

El proyecto, a lo largo de todos estos años, ha recopilado información muy valiosa en torno a las inquictudes, preocupaciones y actitudes de los docentes, lo que ha permitido identificar aquellas áreas medulares en las cuales los académicos universitarios requieren actualización, como es el caso de las temáticas relacionadas con discapacidad: terminología específica, caracterización de las distintas discapacidades, legislación existente y políticas institucionales. Además, existe un gran interés por capacitación acerca de cómo atender las necesidades educativas especiales a nivel universitario, las estrategias pedagógicas y didácticas requeridas, así como posibles adecuaciones y adaptaciones a aplicar. 


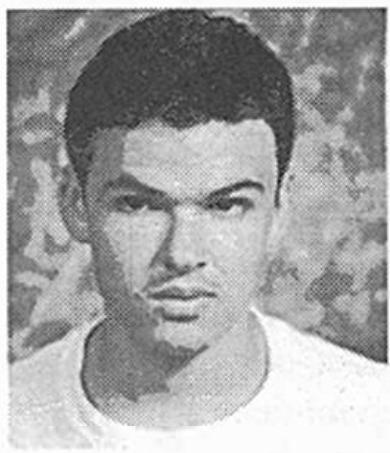

"Desde que ingresé a la Universidad en cl año 2002, me puse en contacto con el proyecto, solicitando la ayuda de lectores que me asisticran en las matcrias que curso. La experiencia ha sido bastante gratificante, pues los lectores en una gran mayoría, han sido personas responsables y tiencn una clara forma de lectura y de intcrpretación de palabras.

Gracias a cstc proyecto con cl que cuenta la Universidad Nacional, sc nos facilita el mćtodo de estudioya que nos da las herramientas indispensables, como las computadoras adaptadas con cl programa JAWS y el cscáner, que son de gran utilidad para todos nosotros. Estas nos abren una puerta a la información para realizar nuestros trabajos y cstudio de una manera digna. Considero de importancia, se divulgue un programa como éste que vela por nucstros derechos de cstudiante y persona. Invito a que cada vez sea mayor cl número de personas que colaboran con el proyecto y que conozcan los scrvicios que ofrece".

Esteban Segura Peñaranda Estudiante con discapacidad visual

Por otro lado, estc trabajo ha permitido identificar iniciativas propias de los docentes, quiencs han generado estrategias alternativas y creativas para atender las necesidades educativas especiales en el aula universitaria. Algunas de cllas se han dirigido a lograr la accesibilidad a la información, según lo requiera el estudiante durante su proccso de aprendizajc, como por cjemplo, ampliaciones de material impreso, material gráfico en relicve, instrucciones cscritas; apoyos audibles como grabacioncs y descripción oral del material visual presentado. Una experiencia interesante e innovadora tendicnte a lograr la accesibilidad al conocimicnto, la constituyc lo que podemos llamar apoyos conceptuales que se pueden definir como explicacioncs de conceptos abstractos con imágenes litcrarias.

La organización del grupo que rcaliza el propio docentc como apoyo para atender las necesidades de los estudiantes, también se puede constituir en una estratcgia innovadora, que no exige trabajo adicional o paralclo, sino una organización distinta que incluya a todos los alumnos dentro de su propuesta mctodológica. 


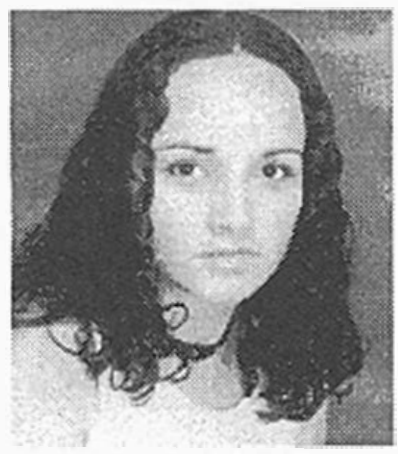

"La experiencia que he obtenido en el proyecto Una educación para todos los alumnos de la UNA. me ha hecho enfientarme con la realidad de que yo, como futura docente, necesito algo más que conocimientos o métodos de enseñanzaaprendizaje. Lo que verdaderamente necesito es abir espacios, a la humildad, al respeto, a la amistad y poner a prueba mi grado de humanidad.

Día a día que dedico mi tiempo para apoyar a un estudiante en sus estudios, hago ejercer mis valores. Además de reconocer que mi labor va más allá de realizar grabaciones, o de digitar materiales, sentarme al lado de otros seres humanos, con los que puedo disfrutar un rato agradable, con quienes además de realizar la labor asignada, aprendo a escuchar, a hablar, a sentir y a darme cuenta de que no estoy sola en el mundo, que existen muchas otras personas que necesitan de mi, como yo de ellas.

Gracias a este arduo trabajo, muchos estudiantes pueden superar los obstáculos que enfrentan para lograr sus sueños. Por esta razón es que mi labor es de mucha satisfacción. Sé que todo mi trabajo, como el de muchas otras personas que apoyan en el proyecto, es base para el futuro de grandes profesionales".

Alicia Zárate Brizuela Estudiante de horas colaboración

Finalmente, es importante destacar que, si bien es indiscutible la tarea por delante que le compete al proyecto Una educación para todos los alumnos de la Universidad Nacional, también es impostergable la participación y el compromiso de toda la institución para lograr la construcción de una Universidad y una sociedad más equitativas y justas para TODOS.

\section{Referencias}

Asamblea Legislativa. (1996). Ley 7600 Igualdad de oportunidades para las personas con discapacidad. San José: Diario Oficial La Gaceta, No. $102 \mathrm{del}$ 29 de mayo de 1996.

Brennan, W.K. (1988). El curriculo para niños con necesidades educativas especiales. Madrid: MEC-Siglo XXI. 
Devalle de Rendo, A. y Vega, V. (1999). Una escuela en y para la diversidad. Buenos Aires: AIQUE.

Herrera. A., Miranda. G. y Zúñiga. E. (1998). Una educación para rodos los alemusos de la Universidad Nacional. Heredia: Universidad Nacional, Vicerrectoria Académica (malcrial impreso).

Heitera, A. y Miranda. G. (2001). UNA: educación de caliclad. Heredia: Universidad Nacional. (matcrial impreso).

Meléndez, L. (2002). De la segregación a la inclusión. El caso de la atención a las personas con necesidades educarivas especiales en Costa Rica. San José: MEP.

Rodríguč, M., Jiménez, F., Benavides, Y., Miranda, G., Torres, A. C., Vargas, E., Zúñiga, E., Cárdenas, F., Chacón, E. y Delgado, R. (1994). Plan de atención global del estudiante con discapacidad. Heredia: Universidad Nacional, Vicerrecioria de Vida Estudiantil, Comisión institucional para la atención de estudiantes con discapacidad.

Sánche\%, A. y Torres, J. (1997). De la educación especial a las necesidades educativas expeciales: aproximación histórica, marco concepunal )' legislarivo. Madrid: Edicioncs Pirámide.

UNESCO (1990). Declaración mundial de educación para todos. Jomtien, Tailandia: UNESCO.

Universidad Nacional. (1993). Estanuro organico. Heredia, Cosla Rica: Departamento de Publicaciones.

Van Steenlandt, D. (1991). La insegración de niños discapacirados a la eclucación comin. Santiago: Editorial UNESCO/OREALC. 